\title{
Lymphatic and blood vessel density in the follicular patterned lesions of thyroid
}

\author{
Tamar A Giorgadze ${ }^{1}$, Zubair W Baloch ${ }^{2}$, Teresa Pasha ${ }^{2}$, Paul J Zhang ${ }^{2}$ and \\ Virginia A LiVolsi \\ ${ }^{1}$ Department of Pathology, East Tennessee State University, Johnson City, TN, USA and ${ }^{2}$ Department of \\ Pathology and Laboratory Medicine, University of Pennsylvania, Medical Center, Philadelphia, PA, USA
}

\begin{abstract}
The histologic distinction of follicular patterned lesions of thyroid, that is follicular adenoma, follicular carcinoma, and the follicular variant of papillary thyroid carcinoma can be extremely difficult. The differential diagnostic criteria regarding nuclear features of papillary thyroid carcinoma are subjective, resulting in high interobserver variability. Although papillary thyroid carcinoma metastasizes mainly via lymphatic vessels, whereas follicular carcinoma spreads mostly hematogenously, there are no data regarding utility of objective quantitative criteria such as lymphatic and general blood vessel density for the differential diagnosis of these lesions. In this study, 35 follicular patterned lesions of thyroid (14 follicular adenomas, 10 follicular carcinomas, and 11 of the follicular variant of papillary thyroid carcinomas) were evaluated immunohistochemically. An assessment of intra- and peritumoral lymphatic vessel density was performed using novel lymphatic endothelium-specific marker D2-40, and the intra- and peritumoral general vessel density was determined by the panendothelial marker CD31. There were no significant differences in the intra- and/or peritumoral general vessel densities, and peritumoral lymphatic vessel densities among follicular adenoma, follicular carcinoma and the follicular variant of papillary thyroid carcinoma. In contrast, the intratumoral lymphatic vessel density was significantly higher in the follicular variant of papillary thyroid carcinoma than in either follicular adenoma or follicular carcinoma $(34.63,15.04$, and 0.11 respectively; $P<0.0001)$. The results of the study show that intratumoral lymphatic vessel density may serve as a useful tool in the differential diagnosis of follicular patterned lesions of thyroid.

Modern Pathology (2005) 18, 1424-1431. doi:10.1038/modpathol.3800452; published online 27 May 2005
\end{abstract}

Keywords: follicular patterned lesions of thyroid; lymphatic vessel density; general vessel density; immunohistochemistry; differential diagnosis

The differential diagnosis of follicular patterned lesions of thyroid such as follicular adenoma, follicular carcinoma, and the follicular variant of papillary thyroid carcinoma is one of the cornerstone problems of thyroid pathology. The existing diagnostic criteria regarding nuclear features of papillary thyroid carcinoma are subjective and cause high interobserver variability even among the experts, especially if these features are not fully developed or are expressed focally. ${ }^{1}$ None of the immunohistochemical markers or other ancillary studies currently used for the differential diagnosis of these lesions proved to be specific.

Correspondence: Dr TA Giorgadze, MD, PhD, Department of Pathology, East Tennessee State University, PO Box 70568, Johnson City, TN 37604, USA.

E-mail: giorgadz@etsu.edu

This work was presented in abstract form at the International Academy of Pathology Congress in Brisbane, Australia, October 13,2004

Received 17 January 2005; revised 21 April 2005; accepted 22 April 2005; published online 27 May 2005
It is known that papillary thyroid carcinomas have a propensity for intrathyroidal spread via lymphatic vessels with further lymphagenous metastases to the regional lymph nodes, whereas follicular carcinomas metastasize mainly hematogenously. These peculiarities in the metastatic features of different thyroid tumors are not fully understood, but might be related to tumor angiogenesis as a major factor in tumor development and progression. Recent reports on thyroid tumor angiogenesis have shown that the expression of different factors promoting de novo blood vessel formation (hemangiogenesis) is increased in all thyroid tumors, whereas the expression of lymphangiogenic growth factors is significantly increased only in papillary thyroid carcinomas. ${ }^{2-5}$ The above-mentioned data prompted us to hypothesize that objective morphometric criteria reflecting both hemangiogenesis and lymphangiogenesis, such as microvessel density, could be used in the differential diagnosis of thyroid tumors in general, and follicular patterned lesions of thyroid in particular. 
To date, in contrast to numerous studies on general blood vessel density in thyroid neoplasms, ${ }^{6-13}$ there are no reports in the English literature regarding lymphatic vessel density in thyroid tumors other than papillary thyroid carcinoma. ${ }^{14}$

The goals of our study were to evaluate tumor lymphangiogenesis in follicular patterned lesions of thyroid and the utility of objective quantitative criteria such as lymphatic and general blood vessel density for the differential diagnosis of these lesions. For this purpose, in a series of follicular patterned lesions of thyroid, we quantitatively evaluated the intra- and peritumoral lymphatic vessel density using the novel lymphatic endothelium specific immunohistochemical marker D2-40, and compared it to intra- and peritumoral general vessel density as determined by the panendothelial marker CD31.

\section{Materials and methods}

In total, 35 cases of follicular patterned lesions of thyroid (14 follicular adenomas, 10 follicular carcinomas, and 11 of the follicular variant of papillary thyroid carcinoma) were retrieved from personal consultation files of one of the authors (VAL). Clinicopathological data were reviewed when available. Representative formalin-fixed, paraffin-embedded tissue sections were selected for each case after a histologic review of hematoxylin- and eosinstained slides. Immunohistochemical stains were performed on 5 - $\mu \mathrm{m}$-thick sections. A mouse monoclonal antibody, clone D2-40 (Signet Laboratories, Dedham, MA, USA; dilution 1:25) was used. Prior to antibody incubation, sections were boiled in $1 \times$ ethylenediaminetetraacetic acid buffer (Laboratory Vision) for $20 \mathrm{~min}$. A section of each case was also stained with anti-CD31 (clone JC70A, 1:10; DakoCytomation Corporation, Carpinteria, CA, USA). The pretreatment for CD31 was enzyme digestion in $0.06 \%$ pronase for $15 \mathrm{~min}$ at $37^{\circ} \mathrm{C}$. Immunohistochemical staining was performed in DakoCytomation Autostainer ${ }^{\mathrm{TM}}$ with the EnVision + horseradish peroxidase diaminobenzidine for mouse (DakoCytomation). Normal mouse serum (1:1000) was used to substitute primary antibody in each case, as negative control. The peri- and intratumoral vessel density was determined in five high-power fields $(\times 400)$ of the tissue sections in areas with the highest vascularity (hot spots) as previously described, ${ }^{12}$ and the results were recorded as the sum of all vessel counts per five high-power fields $(\times 400)$. Intratumoral vessels were defined as vessels within the tumor cell-occupied area. Peritumoral vessels were defined as vessels within one high-power field $(\times 400)$ around the tumor cell-occupied area or beyond the tumor capsule. Only structures that morphologically appeared as vascular and stained with either immunomarker were taken into account. Vessels were counted independently by two patho- logists (TG and ZB, or TG and VAL) in each case. The final score for each case was determined as a mean of the values determined from two different readings of intra- and/or peritumoral vessel densities.

Mean values, standard deviations, and standard errors of mean were calculated for each group of tumors. Mean lymphatic and general vascular densities were compared using the one way ANOVA (analysis of variance) test. Statistical significance was determined by $P$-value $(<0.0001)$. Spearman's correlation coefficients were obtained in cases when statistically significant differences in vascular densities were found.

\section{Results}

Immunohistochemical staining for D2-40 highlighted intra- and peritumoral lymphatic vessels as well as lymphatics in non-neoplastic thyroid parenchyma. Blood vessels that were surrounded by smooth muscle and contained red blood cells were completely negative for this marker, whereas vascular structures morphologically consistent with lymphatics with lymphocytes in them showed strong positivity for D2-40 and served as an excellent positive internal control (Figure 1a and b, arrow).

While evaluating intratumoral lymphatic vessel density in the follicular variant of papillary thyroid carcinomas, an increased number of small lymphatic vessels with both opened and collapsed lumina could be identified in-between the tumor cells, in the fibrous septae separating the lobules of the tumor, adjacent to the tumor capsule, and in the tumor capsule itself (Figure 1c, d, and e). We found an increased number of lymphatic vessels in the areas where, in the corresponding fields of hematoxylin-eosin-stained sections, the nuclear features of papillary thyroid carcinoma were more pronounced, for example, in the areas close to the tumor capsule. Interestingly, islands with an increased number of lymphatic vessels, sharply demarcated from the nontumoral thyroid parenchyma devoid of any lymphatics, could be identified in the areas of intraglandular spread of tumor far away from the main tumor mass (Figure 1f).

In contrast to the follicular variant of papillary thyroid carcinoma, the intratumoral lymphatic vessel density in follicular carcinomas was strikingly low, almost none. In our study, even obviously highly vascular tumors did not show a single lymphatic vessel when evaluated with D2-40 immunostain (Figure $1 \mathrm{~g}$ and $\mathrm{h}$ ). Importantly, one case of follicular patterned lesion with hybrid tumor features that focally showed atypical nuclei suggestive of papillary thyroid carcinoma did not reveal lymphatic vessels, favoring the diagnosis of follicular carcinoma (Figure $1 \mathrm{i}$ and $\mathrm{j}$ ).

There was an increased number of intratumoral lymphatics found in several follicular adenomas, 

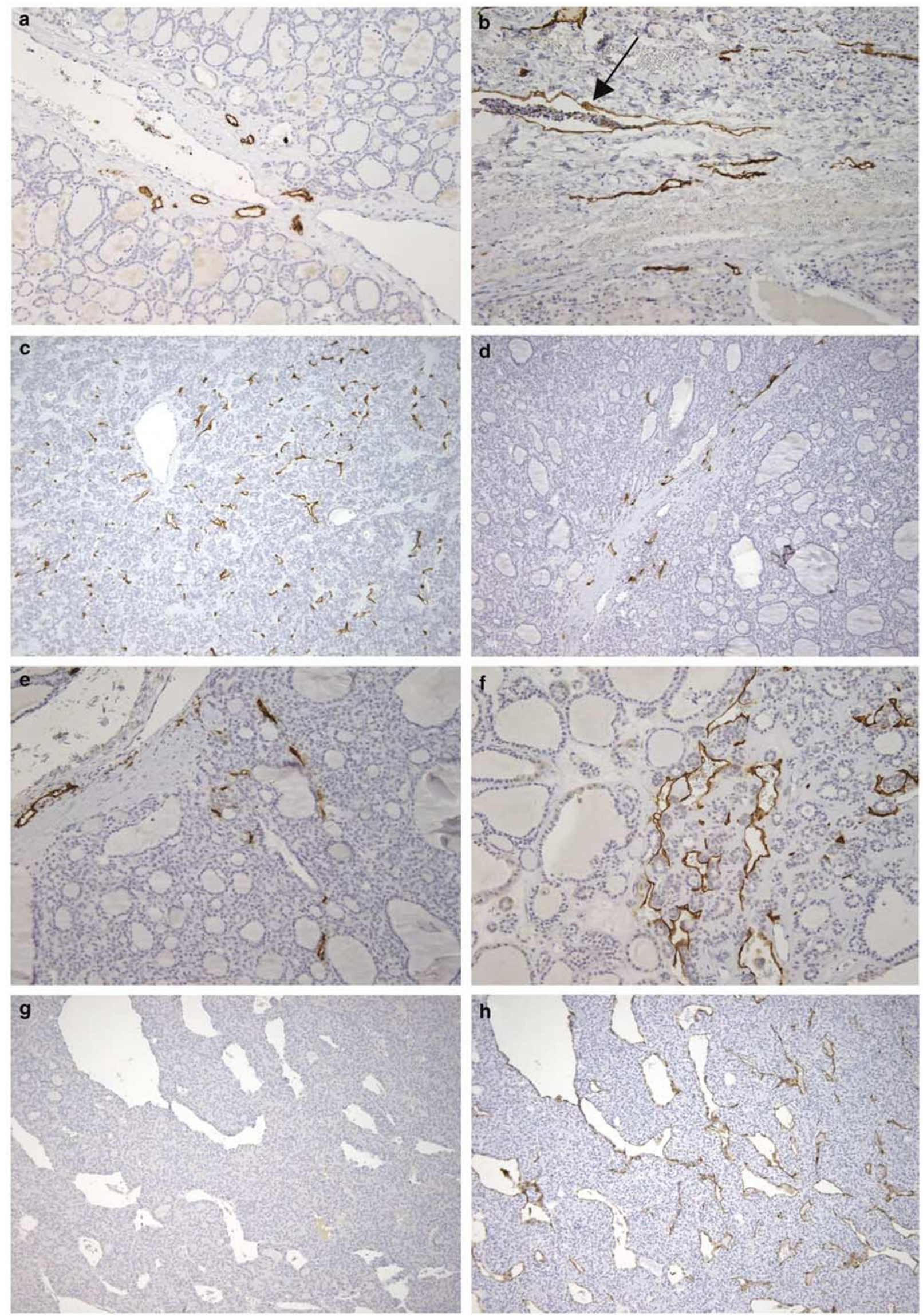
which contributed to the higher mean intratumoral lymphatic vessel density values in follicular adenomas than in follicular carcinomas. In two of these cases, there was a background of severe chronic lymphocytic thyroiditis with an increased number of lymphatic vessels in nontumoral thyroid parenchyma, and in one case, the increased number of lymphatic vessels was in the area of post fine-needle aspiration track (Figure 1k and l).

The quantitative results of intra- and peritumoral lymphatic vessel density in follicular patterned lesions of thyroid are shown in Figure 2a and b. Although hot spots of lymphatic vessels could be identified in follicular adenomas, quantitatively, the intratumoral lymphatic vessel density in the follicular variant of papillary thyroid carcinoma was significantly higher than in both follicular adenoma and follicular carcinoma $(P<0.0001)$. The mean values of intratumoral lymphatic vessel densities were $34.63 \pm 19.02$ (s.e.m.=5.74) for the follicular variant of papillary thyroid carcinoma, $15.04 \pm 18.1$ (s.e.m. $=4.83$ ) for follicular adenoma, and $0.11 \pm 0.3$ (s.e.m. $=0.09$ ) for follicular carcinoma. In our study, large peritumoral lymphatic vessels with open lumina morphologically similar to the lymphatics in non-neoplastic thyroid parenchyma could be found in all follicular patterned lesions of thyroid, and there was no statistically significant difference in the quantity of peritumoral lymphatics among various groups of lesions. The mean values of peritumoral lymphatic vessel density were $39.5 \pm 9.64$ (s.e.m. = 3.05) for the follicular variant of papillary thyroid carcinoma, $37.86 \pm 7.46$ (s.e.m. $=1.99$ ) for follicular adenoma, and $49.5 \pm 13.51 \quad($ s.e.m. $=4.27)$ for follicular carcinoma (Figure 1m-0).

All thyroid tumors in our study showed high intra- and peritumoral general blood vessel density highlighted by CD31 immunostain (Figure 1p-u, respectively). The mean values of intratumoral general vessel densities were $166.5 \pm 29.04$ (s.e.m. $=$ 11.9) for the follicular variant of papillary thyroid carcinoma, $262.62 \pm 71.18$ (s.e.m. $=25.24$ ) for follicular adenoma and $235.57 \pm 153.19$ (s.e.m. $=58.02$ ) for follicular carcinoma. Although intratumoral general vessel density in follicular adenoma and follicular carcinoma seemed to be somewhat higher than in the follicular variant of papillary thyroid carcinoma, the difference did not reach statistical significance. Nor was there significant difference in peritumoral general vessel densities. The mean values of peritumoral general vessel densities were 134.6 \pm 47.62 for the follicular variant of papillary thyroid carcinoma (s.e.m. $=21.35$ ), $126.5 \pm 47.6$ (s.e.m. $=16.8$ ) for follicular adenoma, and 141.71 \pm 32.11 (s.e.m. $=12.16$ ) for follicular carcinoma (Figure 2c and d).

\section{Discussion}

Angiogenesis plays a crucial role in solid tumor growth, progression, invasion, and metastasis. Tumor growth beyond a critical size requires de novo blood vessel formation. Numerous factors both promoting and inhibiting angiogenesis have been identified. Very little is known about what modulates angiogenic switch in the tumors and how the tumor cells gain access to the vascular structures for further dissemination-through the expansion and invasion of pre-existing vessels or by de novo tumor angiogenesis. ${ }^{15,16}$ On the other hand, from multiple clinical observations, it is clear that there definitely are human malignancies for which the most common pathway of initial dissemination is lymphagenous rather than hematogenous spread. Papillary thyroid carcinoma is one of them.

Angiogenesis in thyroid neoplasm has been studied extensively. ${ }^{2,3}$ Most of these studies reflect the level of expression of different angiogenesis factors and microvessel density in thyroid tumors, and correlation of these factors with clinicopathological features including thyroid tumor histotype, differentiation, presence of regional lymph node, and distant metastases and prognosis., ${ }^{2,3,17}$

Among angiogenic factors most extensively evaluated in thyroid neoplasms is vascular endothelial growth factor (VEGF), one of the major regulators of tumor angiogenesis. VEGF gene and protein expression have been shown to be increased not only in thyroid tumors, but in Graves' disease, Hashimoto thyroiditis and goiter. ${ }^{2,3,17-21}$

The intensity of VEGF expression has been shown to be associated with thyroid tumor behavior. Higher

Figure 1 Lymphatic and blood vessels in the follicular patterned lesions of thyroid. (a) D2-40-positive intratumoral lymphatic vessels with open and collapsed lumina; note the absence of staining in the adjacent blood vessels. (b) Immunostain for D2-40 highlighting nonneoplastic thyroid tissue with peritumoral lymphatic vessels containing lymphocytes (arrow). (c-e) D2-40 immunostain in the follicular variant of papillary thyroid carcinoma highlighting small irregularly shaped lymphatic vessels in-between the tumor cells (c), in the intratumoral fibrous septae (d), close to the tumor capsule and in the tumor capsule (e). (f) Numerous morphologically abnormal lymphatic vessels highlighted by D2-40 in the area of intraglandular spread of the follicular variant of papillary thyroid carcinoma; note the absence of lymphatic vessels in the adjacent thyroid parenchyma. (g) Section of follicular carcinoma demonstrating absence of immunostain for D2-40. (h) Same field of follicular carcinoma as in (g). Numerous blood vessels highlighted by CD31 immunostain. (i) Hematoxylin-eosin-stained section of hybrid tumor with atypical nuclei (inset). (j) Same tumor as in (i). Complete absence of lymphatic vessels with D2-40 staining. (k) Hematoxylin-eosin-stained section of follicular adenoma with fine-needle aspiration track. (l) Same field as in (k). D2-40 immunostain demonstrating an increased number of lymphatic vessels in that area. (m-o) Peritumoral lymphatic vessels in the follicular variant of papillary thyroid carcinoma $(\mathbf{m})$, follicular carcinoma (n), and follicular adenoma (o). (p-r) Intratumoral blood vessels in the follicular variant of papillary thyroid carcinoma (p), follicular carcinoma (q), and follicular adenoma (r). (s-u) Peritumoral blood vessels in the follicular variant of papillary thyroid carcinoma (s), follicular carcinoma (t), and follicular adenoma (u). 

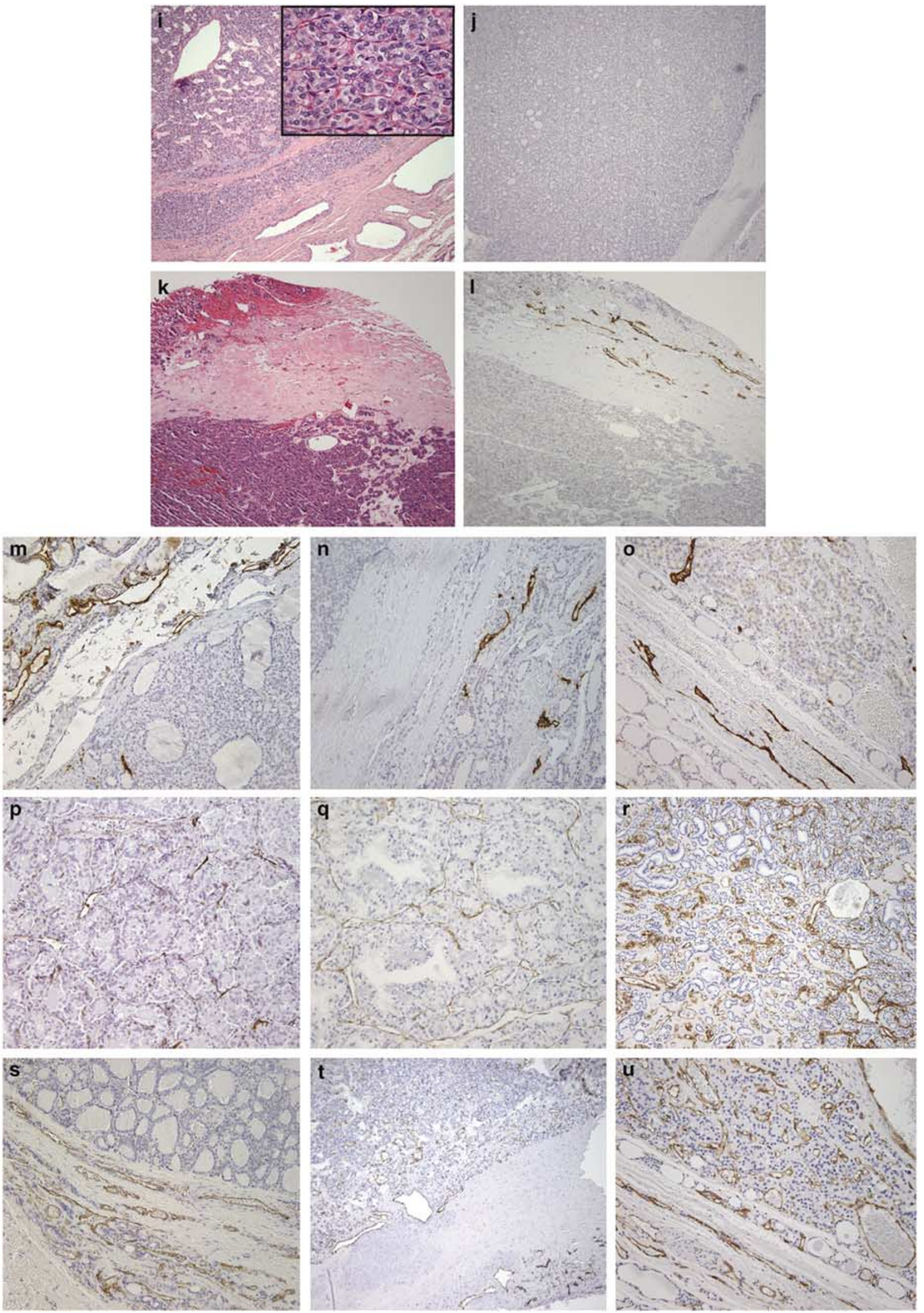

Figure 1 Continued. 


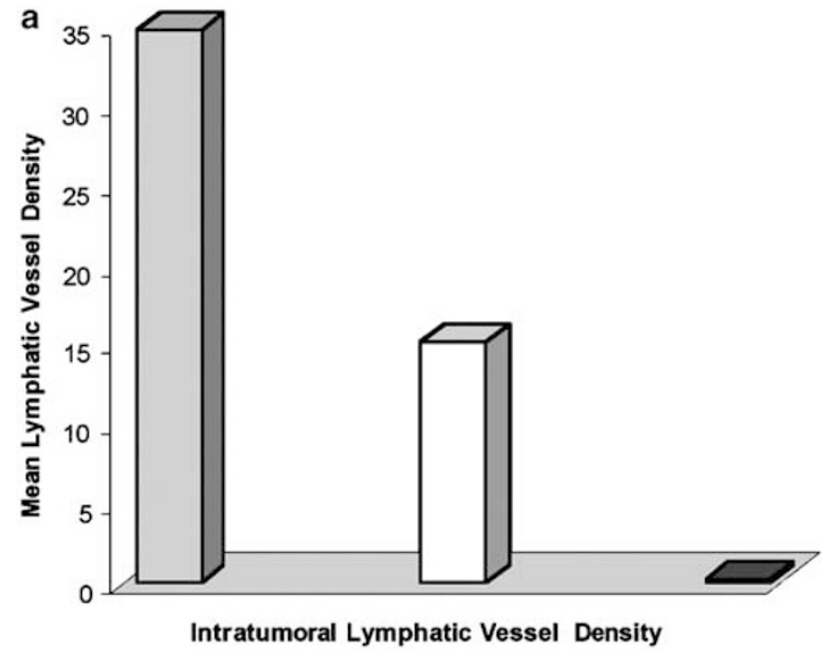

口 Follicular Variant of Papillary Thyroid Carcinoma a Follicular Adenoma

- Follicular Carcinoma

C

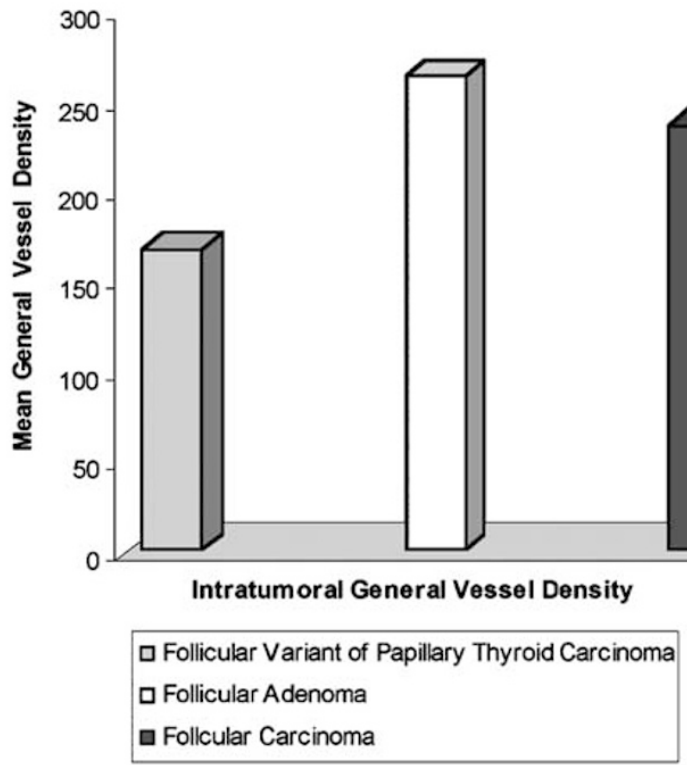

b
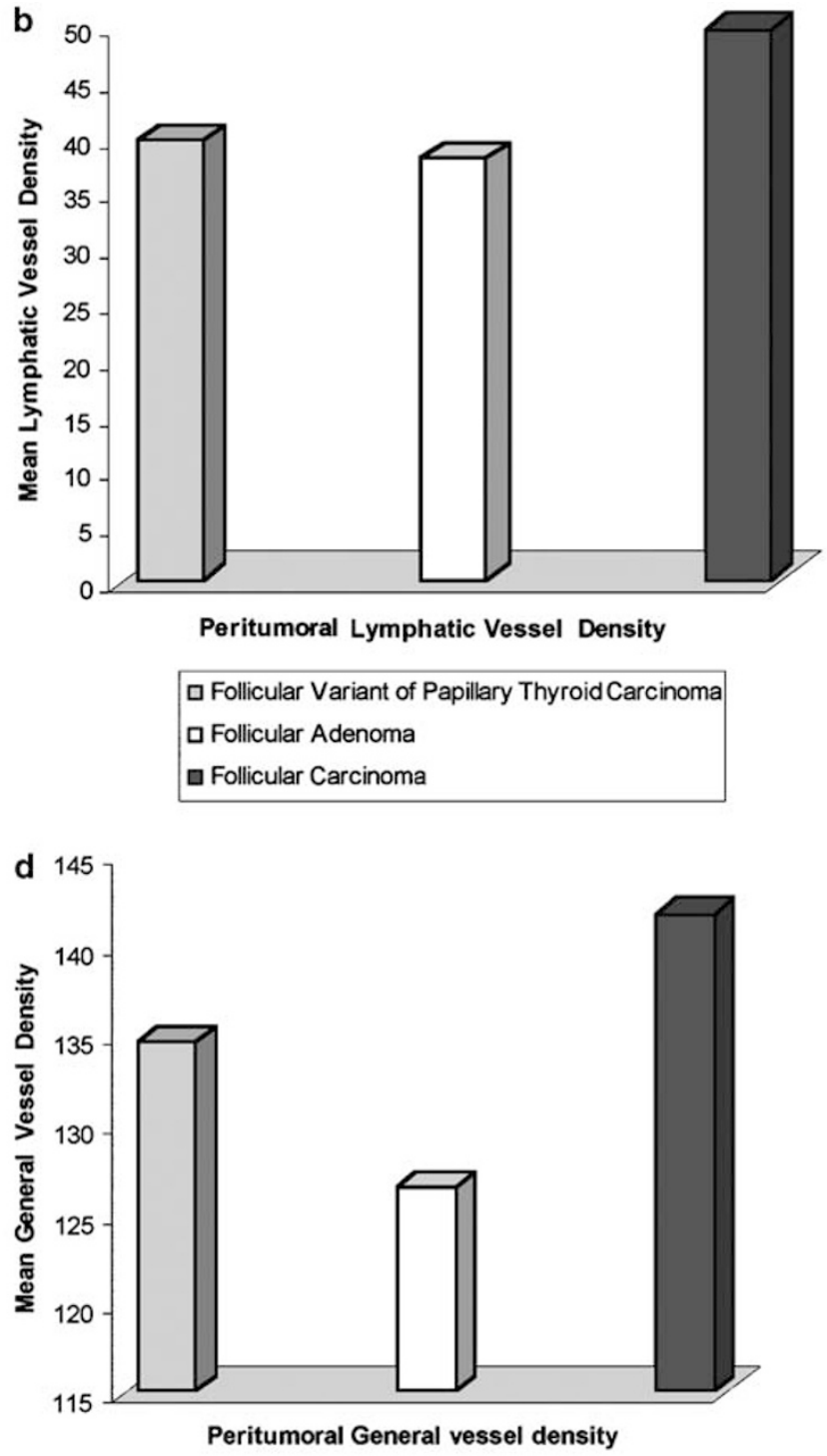

口 Follicular Variant of Papillary Thyroid Carcinoma 口 Follicular Adenoma

- Follicular Carcinoma

Figure 2 Intra-and peritumoral lymphatic vessel density and general vessel density in the follicular patterned lesions of thyroid. (a) Intratumoral lymphatic vessel density. (b) Peritumoral lymphatic vessel density. (c) Intratumoral general vessel density. (d) Peritumoral general vessel density.

VEGF expression is present in metastatic thyroid cancer than in nonmetastatic disease, correlates with the tumor size and is associated with increased risk of recurrence and decreased disease-free survival in papillary thyroid carcinomas. ${ }^{3,20,22}$ Increased VEGF concentration in papillary and follicular thyroid carinomas parallels with the cell proliferation activity evaluated by immunohistochemical stain for Ki-67, indicating increased angiogenesis in both types of tumors. ${ }^{20}$

Members of the VEGF family have been shown to promote both hemangiogenesis and lymphangiogenesis in the thyroid during disease processes.
Recently, it has been demonstrated that levels of VEGF-C, which stimulates lymphangiogenesis, are increased in PTC, but not in the other thyroid cancers prone to metastasize via lymphatics. ${ }^{4,5}$

While there is a clear relationship between VEGF expression and thyroid tumor behavior, the data regarding the significance of microvessel density in thyroid neoplasms are rather confusing. Thyroid tumors are more vascular than the normal thyroid gland. Increased microvessel density was found in malignant vs benign follicular tumors, well-differentiated thyroid carcinomas, and papillary thyroid carcinomas. ${ }^{6-8,10,12}$ Surprisingly, in some studies, 
reduced microvessel density in papillary thyroid carcinomas was associated with poor differentiation, worse prognosis, and reduced survival. ${ }^{6,12}$ In contrast, in other studies, an increased risk of recurrence, worse prognosis, and poorer survival were associated with increased microvessel density, ${ }^{9,11,13}$ whereas Fontanini et $a l^{7}$ found association of increased microvessel density with poor prognosis only for medullary, but not for papillary or follicular thyroid carcinomas.

There could be many methodological reasons for these controversies, including selection of vascular immunohistochemical markers (CD31, CD34, or Factor VIII), methods of counting vessels, observer variability, and most importantly, no discrimination of blood vessels from lymphatic vessels by immunohistochemical markers used in these studies. The latter explains the fact that despite numerous publications regarding microvessel density, there is only one recent report regarding lymphatic vessel density in thyroid tumors, and specifically in well-differentiated papillary thyroid carcinomas. ${ }^{14}$ In this report, Hall et a ${ }^{14}$ showed that not only are there numerous morphologically abnormal intratumoral lymphatics in papillary thyroid carcinomas, but also the development of intratumoral lymphatics appears to be associated with multifocal disease and presence of lymph node metastases at presentation.

The immunohistochemical studies regarding lymphangiogenesis and lymphatic vessel density in tumors became possible only after the recent discovery of lymphatic endothelium-specific markers such as LYVE-1, podoplanin, and D2-40. The monoclonal antibody D2-40 was introduced most recently. This antibody has been thoroughly studied in our laboratory and proved to be useful for both qualitative and quantitative assessment of lymphatic vessels in different tumors. ${ }^{23,24}$

In the present study, we used D2-40 for the assessment of lymphatic vessel density in the follicular patterned lesions of thyroid. In order to evaluate lymphangiogenesis in these lesions and the utility of objective quantitative criteria such as lymphatic and general blood vessel density in their differential diagnosis, we determined the intra- and peritumoral lymphatic vessel density using D2-40 antibody, and compared it to intra- and peritumoral general vessel density determined by the panvascular marker CD31.

Our study showed that the intratumoral lymphatic vessel density in the follicular variant of papillary thyroid carcinoma is significantly higher than in follicular adenoma and follicular carcinoma. Spearman's correlation coefficient was 0.92 $(<0.0001)$ for the follicular variant of papillary thyroid carcinoma, $0.95(<0.0001)$ for follicular adenoma, and $1.0(<0.0001)$ for follicular carcinoma. The difference was especially striking when compared with lymphatic vessel density in follicular carcinomas, in which intratumoral lymphatics were almost nonexistent. Although overall lymphatic vessel density in follicular adenoma, quantitatively, was significantly lower than in the follicular variant of papillary thyroid carcinoma, there were several follicular adenomas with hot spots of lymphatic vessels. In one of them, the increased number of lymphatics was seen in the fine-needle aspiration tract, and in two others, there was a background of severe chronic lymphocytic thyroiditis. We do not have enough data to suggest that inflammatory cells of background thyroiditis might somehow promote lymphangiogenesis in thyroid tumors, but that could be a possible explanation. We also found an increased number of lymphatic vessels in several adenomatous nodules adjacent to the follicular variant of papillary thyroid carcinoma. Again, we do not have enough data to make any conclusions regarding the lymphatic vessel density in hyperplastic/adenomatous nodules although the work is in progress in our institution (data not shown).

In our study, as in the study by Hall et $a l,{ }^{14}$ the intratumoral lymphatics were morphologically abnormal-small and irregular, suggesting de novo lymphatic vessel formation. Further studies, such as double immunostaining with D2-40 and the proliferative marker Ki-67, could prove active de novo lymphangiogenesis in the thyroid tumors. Hall et $a l^{14}$ also found a significant association between the presence of intratumoral lymphatics and the presence of nodal metastasis at presentation. In the consult cases that we used, we did not have information on lymph nodes and/or follow-up data to perform correlation studies. Nevertheless, the finding of small areas with hot spots of lymphatics away from the main tumor mass raises the possibility that quantitative determination of lymphatic vessel density can be potentially useful in identifying intrathyroidal lymphatic spread, multifocal disease, or even in the diagnosis of papillary microcarcinoma. In our study, we did not find significant differences in the intra- and peritumoral general vessel density among various follicular patterned lesions of thyroid. This can be explained by the fact that all thyroid tumors, both benign and malignant, are highly vascular. We believe that it is the de novo intratumoral lymphatic vessel formation that determines the biological behavior and aggressiveness of papillary thyroid carcinoma, and that the assessment of intratumoral lymphatic vessel density can be used as an objective criterion in distinction of the follicular variant of papillary thyroid carcinoma from other follicular patterned lesions of thyroid.

\section{References}

1 Lloyd RV, Erickson LA, Casey MB, et al. Observer variation in the diagnosis of follicular variant of papillary thyroid carcinoma. Am J Surg Pathol 2004; 28:1336-1340. 
2 Ramsden JD. Angiogenesis in the thyroid gland. J Endocrinol 2000;166:475-480.

3 Turner HE, Harris AL, Melmed S, et al. Angiogenesis in endocrine tumors. Endocr Rev 2003;24:600-632.

4 Fellmer PT, Sato K, Tanaka R, et al. Vascular endothelial growth factor-C gene expression in papillary and follicular thyroid carcinomas. Surgery 1999;126:1056-1061.

5 Hung CJ, Ginzinger DG, Zarnegar R, et al. Expression of vascular endothelial growth factor- $\mathrm{C}$ in benign and malignant thyroid tumors. J Clin Endocrinol Metab 2003;88:3694-3699.

6 Herrmann G, Schumm-Draeger PM, Muller C, et al. T lymphocytes, CD68-positive cells and vascularisation in thyroid carcinomas. J Cancer Res Clin Oncol 1994; 120:651-656.

7 Fontanini G, Vignati S, Pacini F, et al. Microvessel count: an indicator of poor outcome in medullary thyroid carcinoma but not in other types of thyroid carcinoma. Mod Pathol 1996;9:636-641.

8 Segal K, Shpitzer T, Feinmesser M, et al. Angiogenesis in follicular tumors of the thyroid. J Surg Oncol 1996; 63:95-98.

9 Ishiwata T, Iino Y, Takei H, et al. Tumor angiogenesis as an independent prognostic indicator in human papillary thyroid carcinoma. Oncol Rep 1998;5:13431348.

10 Goldenberg JD, Portugal LG, Wenig BL, et al. Well-differentiated thyroid carcinomas: p53 mutation status and microvessel density. Head Neck 1998;20: 152-158.

11 Dhar DK, Kubota H, Kotoh T, et al. Tumor vascularity predicts recurrence in differentiated thyroid carcinoma. Am J Surg 1998;176:442-447.

12 Akslen LA, LiVolsi VA. Increased angiogenesis in papillary thyroid carcinoma but lack of prognostic importance. Hum Pathol 2000;31:439-442.

13 Tanaka K, Sonoo H, Kurebayashi J, et al. Inhibition of infiltration and angiogenesis by thrombospondin-1 in papillary thyroid carcinoma. Clin Cancer Res 2002; 8:1125-1131.
14 Hall FT, Freeman JL, Asa SL, et al. Intratumoral lymphatics and lymph node metastases in papillary thyroid carcinoma. Arch Otolaryngol Head Neck Surg 2003;129:716-719.

15 Fox SB. Tumour angiogenesis and prognosis. Histopathology 1997;30:294-301.

16 Pepper MS. Lymphangiogenesis and tumor metastasis: myth or reality? Clin Cancer Res 2001;7:462-468.

17 Bunone G, Vigneri P, Mariani L, et al. Expression of angiogenesis stimulators and inhibitors in human thyroid tumors and correlation with clinical pathological features. Am J Pathol 1999;155:1967-1976.

18 Soh EY, Duh QY, Sobhi SA, et al. Vascular endothelial growth factor expression is higher in differentiated thyroid cancer than in normal or benign thyroid. J Clin Endocrinol Metab 1997;82:3741-3747.

19 Iitaka M, Miura S, Yamanaka K, et al. Increased serum vascular endothelial growth factor levels and intrathyroidal vascular area in patients with Graves' disease and Hashimoto's thyroiditis. J Clin Endocrinol Metab 1998;83:3908-3912.

20 Klein M, Picard E, Vignaud JM, et al. Vascular endothelial growth factor gene and protein: strong expression in thyroiditis and thyroid carcinoma. J Endocrinol 1999;161:41-49.

21 Kilicarslan AB, Ogus M, Arici C, et al. Clinical importance of vascular endothelial growth factor (VEGF) for papillary thyroid carcinomas. APMIS 2003; 111:439-443.

22 Lennard CM, Patel A, Wilson J, et al. Intensity of vascular endothelial growth factor expression is associated with increased risk of recurrence and decreased disease-free survival in papillary thyroid cancer. Surgery 2001;129:552-558.

23 Giorgadze TA, Zhang PJ, Pasha T, et al. Lymphatic vessel density is significantly increased in melanoma. J Cutan Pathol 2004;31:672-677.

24 Dumoff KL, Chu C, Xu X, et al. Low D2-40 immunoreactivity correlates with lymphatic invasion and nodal metastasis in early-stage squamous cell carcinoma of the uterine cervix. Mod Pathol 2005;18:97-104. 